\title{
Providing Location Everywhere
}

\author{
Ricardo Anacleto *, Lino Figueiredo, Paulo Novais, and Ana Almeida \\ GECAD - Knowledge Engineering and Decision Support, \\ R. Dr. Antonio Bernardino de Almeida, 431. \\ 4200-072 Porto, Portugal \\ $\{$ rmao, lbf, amn\}@isep.ipp.pt \\ pjon@di.uminho.pt
}

\begin{abstract}
The ability to locate an individual is an essential part of many applications, specially the mobile ones. Obtaining this location in an open environment is relatively simple through GPS (Global Positioning System), but indoors or even in dense environments this type of location system doesn't provide a good accuracy. There are already systems that try to suppress these limitations, but most of them need the existence of a structured environment to work. Since Inertial Navigation Systems (INS) try to suppress the need of a structured environment we propose an INS based on Micro Electrical Mechanical Systems (MEMS) that is capable of, in real time, compute the position of an individual everywhere.
\end{abstract}

Keywords: Location Systems, Dead Reckoning, GPS, MEMS, Machine Learning, Optimization

\section{Introduction}

Location information is an important source of context for ubiquitous computing systems. Using this information, applications can provide richer, more productive and more rewarding user experiences. Also, location-awareness brings many possibilities that make mobile devices even more effective and convenient, at work and in leisure.

The ability of mobile applications to locate an individual can be exploited in order to provide information to help or to assist in decision-making.Some examples include electronic systems to help people with visual impairments [10], support systems for a tourist guide at an exhibition [5] and navigation systems for armies [7] [19].

In an open environment we use GPS (Global Positioning System) to retrieve users' location with good accuracy. However, indoors or in a more dense environment (big cities with tall buildings, dense forests, etc) GPS doesn't work or

\footnotetext{
* The authors would like to acknowledge FCT, FEDER, POCTI, POSI, POCI and POSC for their support to GECAD unit, and the project PSIS (PTDC/TRA/72152/2006).
} 
doesn't provide satisfactory accuracy. Consequently, location-aware applications sometimes don't have access to the user location.

The aim of this work is to design a system capable of, in real time, determining the position of an individual (preferably in a non-structured environment), where GPS is not capable.

The motivation for this project comes from previous publications, where a recommendation system to support a tourist when he goes on vacations, was presented [1] [2]. Mobile devices currently available on the market already have built-in GPS, which provides the necessary location context to recommend places of interest and to aid the tourist in planning trip visits [17]. However, this assistance, with current technology, can only be used in environments with GPS signal. In closed or dense environments the system can't easily retrieve the user location context. For example, in an art gallery if the system knows the tourist current position inside the building, it can recommend artworks to view and learn even more about the tourist tastes.

To remove this limitation, a system that provides precise indoor location of people becomes necessary. Actually, there are already some proposed systems that retrieve indoor location with good precision. However most of these solutions force the existence of a structured environment. This can be a possible solution when GPS isn't available, but only indoors. In a dense forest we don't have this kind of systems. To retrieve the location on this type of terrain can be very useful for position knowledge of a fireman's team. Also, the implementation of a structured environment using this type of technologies is very expensive and it becomes unfeasible to incorporate this type of systems in all the buildings of the world.

Since location without using a structured environment remains an open research problem, the main goal of the proposed project is to minimize deployment and infrastructure costs and provide location everywhere.

As users of these types of devices (and applications) are on foot, the INS is one of the most appropriate solutions to be used. This system consists of Micro Electrical Mechanical Systems (MEMS) devices, which can be accelerometers, gyroscopes and other types of sensors. MEMS are small in size, which allows easy integration into clothing. Usually, they communicate with a central module using a wireless network (e.g., Bluetooth). These devices obtain individual movements information independently of the building infrastructure. All this sensory set requires the implementation of a sensor fusion. This includes algorithms that can interpret the sensors information and thereby determine the individual position. The collected information, in addition to the motion speed and direction, must also be able to determine the step width and the individual position (sitting, lying or standing).

This type of system uses the PDR (Pedestrian Dead Reckoning) technique [4]. PDR normally is composed by three key technologies: tracking of the sensor's behavior, walking locomotion detection and walking velocity estimation. But unfortunately, large deviations of these sensors can affect performance, as well 
as the various forms in which a human can move, so this is the project's biggest challenge: to correct the sensor deviations.

A module working only with PDR is not able to ensure that the geographical positions are accurate within a few meters. In fact, although these deviations may be small for every millisecond, the positioning error caused by a sustainable use of the system can exceed one meter in 10 seconds [23].

In order to provide a better contextualization of the existing work on the field, Chapter 2 gives a brief description of the most relevant INS and indoor location systems. In Chapter 3 we present our main objectives to implement an INS and how we intend to complete them, presenting the main challenges and the methodology we will use to suppress them. Finally, Chapter 4 presents some conclusions regarding the presented problem.

\section{State of the Art}

Indoor localization technologies hold promise for many ambient intelligence applications to facilitate, for example, decision-making. However, many of the existent systems can't work indoors or in too dense environments. This happens because they work based on the location obtained by GPS.

Several localization techniques already exist to give indoor positioning, but some of them doesn't provide good accuracy or are too difficult to implement. From the selected articles we have divided them into two main categories based on their localization techniques: Radio Frequency Waves and Pedestrian Dead Reckoning.

Radio frequency waves are solutions that estimate the location of a mobile target in the environment by measuring one or more properties of an electromagnetic wave radiated by a transmitter and received by a mobile station. These properties typically depend on the distance traveled by the signal and the surrounding environment characteristics. In radio frequency we include technologies such as IEEE 802.11, Infrared, Bluetooth and Radio-Frequency IDentification (RFID) tags.

As examples of these systems we have: RADAR (Wireless LAN) [3], Active Badge (Infrared) [25], a home localization system (Bluetooth) [12], a project for the location of objects and people using RFID tags [22], among others [11]. The main problem with these systems is that they need a structured environment in order to work. This makes them dependent on the particular context and still impractical and expensive.

Pedestrian Dead Reckoning systems use sensors to provide location updates, calculated using information about a previously-estimated location. This position estimation is commonly based on inertial sensors. Since they yield relative positioning information only, an absolute reference is required to specify the displacement reported by an inertial measurement in absolute coordinates.

We have divided the PDR systems into two groups: the pure ones and the hybrid solutions. The first use only inertial sensors to give position (after hav- 
ing a reference point), and the latter use WLAN, Bluetooth and other type of structured indoor location systems to correct the inertial sensors data.

\subsection{Dead Reckoning with Structured Environments}

The solutions based on inertial sensing are subject to big measurement deviations caused by thermal changes in the sensor circuit. This is because the longer the time indoors, the greater will be the diversion from virtual trajectory to the real trajectory. In other words, there is error accumulation and to make a recalibration of the sensors, some projects use a structured environment.

This structured environment can be constituted by surveillance cameras like Kourogi [15] proposes. In his paper two enhancements for PDR performance are introduced: map matching and dynamic estimation of walking parameters. The users' location and orientation are updated by fusing the measurements from the PDR estimation and the maps. The surveillance cameras are used to measure walking velocity in order to recalibrate the sensor parameters. Also a particle filter [18] is used for probabilistic data fusing (based on a Bayesian filter). Probability distribution of the users' location is predicted from the estimated position, orientation and its uncertainties. The results are satisfactory, but the existence of lots of people in the building will, certainly, confuse the system. Another problem is the cost of this system (over $\$ 1500$ ).

Also Wi-Fi signal strength can be used to tackle the traditional drift problems associated with inertial tracking [26]. Woodman proposes a framework constituted by a hip-mounted mobile $\mathrm{PC}$ which is used to log data obtained from a foot-mounted Inertial Measurement Unit (IMU). The IMU contains three orthogonal gyroscopes and accelerometers, which report angular velocity and acceleration respectively. The logs are then post processed on a desktop machine (so it isn't a real-time location system). Like Kourogi's system, this uses Bayesian filters to probabilistically estimate the state of a dynamic system based on noisy measurements. The particle filter update consists of three steps: Re-sampling, Propagation and Correction. To reduce the cubic-in-time drift problem of the foot-mounted IMU, they apply a Zero Velocity Update (ZVU) [27], in which the known direction of acceleration due to gravity is used to correct tilt errors which are accumulated during the previous step. Besides Wi-Fi, it also uses a map that acts like a collection of planar floor polygons. The system obtains the Received Signal Strength Indication (RSSI) information by querying the Wi-Fi hardware. Each query returns a list of visible Wi-Fi access points and corresponding RSSI measurements. Each floor polygon corresponds to a surface in the building on which a pedestrians foot may be grounded. Each edge of a floor polygon is either an impassable wall or a connection to the edge of another polygon. Then the system tries to make a correspondence between the Wi-Fi signals, the map and the IMU. A system evaluation was performed in a three floor building, with a total area of $8725 \mathrm{~m}^{2}$, and the error proved to be $0.73 \mathrm{~m}$ in $95 \%$ of the time.

The system proposed by Evennou and Marx [8], it is also based on an IEEE 802.11 wireless network. The absolute positional information obtained from the wireless network was combined with the relative displacements and rotations 
reported by a gyroscope, a dual-axis accelerometer and a pressure sensor. The information obtained from all the sensors was combined through Kalman [21] and particle filtering. To calculate the user displacement, an accelerometer was used to count the number of steps taken. Then, a constant estimate of the users' step length was used to calculate the user total displacement within the environment. The authors showed that combining the information from the bank of sensors yielded improved localization when compared to the usage of each sensor separately. The authors conducted an experiment using their multi-sensor localization system. The localization accuracies reported during such experiment ranged from 1.5 to 3.3 meters.

Renaudin [20] proposes a solution based on RFID tags and inertial MEMS. This system was developed with the intuition to be used by firemen's. MEMS and RFID are hybridized in a structure based on an Extended Kalman Filter and a geographical database. While progressing indoors, the fire fighters deploy RFID tags that are used to correct the large errors affecting MEMS performances. The first team of fire fighters attaches a RFID tag each time it passes a door and when moving from one floor to another, at the beginning and at the end of the stairway. Upon installation, the geographical coordinates of the tag are associated with the tag ID. The RFID tag database is a collection of location coordinates of all the building doors and stairs. This information is then built over the evacuation emergency maps. So, the Extended Kalman Filter uses the 3D coordinates of each detected RFID tag to relocate the trajectory.

The IMU is composed by three sensor modules. The module attached on the shank has a gyroscope, measuring the angular rate of the shank in the sagittal plane, and an accelerometer oriented in the vertical plane that allows the gait analysis. The trunk module contains a triad of gyroscopes, magnetometers and accelerometers providing the orientation information. The thigh module contains an accelerometer measuring the frontal acceleration, which permits posture analysis. The algorithms that compute the route use all the information available in a database to extract the optimum walking path.

The proposed pedestrian navigation solution has been tested on the campus of the "Ecole Polytechnique Federale de Lausanne", in Switzerland. As expected, the PDR position error grows with time, whereas this hybrid positioning solution remains under a certain limit close to 5 meters.

\subsection{Pure Dead Reckoning}

Like we have already seen, there are already systems prepared for indoor positioning. But if we want a system that can provide our location anywhere and doesn't rely in a structured environment, another solution should be used.

One possibility is the use of hybrid solutions based on INS and GPS signal. INS complements the GPS giving location where GPS can't (indoor or in dense environment). The INS uses as start location point the GPS last known coordinate.

As example of an INS system of this type we have NavMote [9] that integrates, MEMS and GPS, in a wireless-device that is in the person's abdominal 
area. This device consists of an accelerometer and a magnetic compass integrated in a generic wireless controller board, with the radio, a processing unit, and power storage all integrated. This device records user movements and stores them in the system. Sensor data are constantly being stored in a 4MB flash memory which allows an operation time of 1.7 hours with a sampling frequency of 30 $\mathrm{Hz}$. Subsequently, the compressed data is transferred to the main system when the wireless-device comes within range of a sensor network (called NetMote). These sensor networks can be spread along the building. On the main system, data are processed into an estimate of the pedestrian trajectory based on a PDR algorithm. The main system is also responsible for trajectory displaying, map matching and other purposes.

This PDR approach uses the acceleration signal pattern to detect the step occurrences and the magnetic compass to provide continuous azimuth information. Based on a simplified kinematic model of a person's gait, the walked distance is provided by summing up the size of each step over the step count. The system showed an error of only 3\%. However, this solution doesn't provide user location in real time. For a 3 minute walk, 45 seconds of download time from the wireless-device to the main system and 25 seconds of system filtration time are typical. So the system is mainly used to track or monitor the user's position with potential security applications rather than to provide high-level interactive travel support.

Walder [24] proposes a system to be used on emergency situations, in large buildings and underground structures. It combines inertial measurements (using 3D accelerometer, gyroscope and 3D magnetometer) of moving persons with building floor plans (CAD drawings) enriched with additional data (e.g., positions of fire extinguishers, room allocation). Because the proposed INS is not accurate enough, the system tries to improve positioning by a permanent interaction between the mobile positioning sensors and floor plans stored in the building information model. The system is composed by four components: position determination, position verification and correction, user interface and communication. The position determination reads raw sensor data and then computes position coordinates. Once computed, a position is verified and corrected, based on the tagged floor plans, if necessary.

The correction of noise and drift is performed by a comparison of real integration with an assumed step length after each step. A normalized step length can be defined as a system parameter, taking into account the user's body size and weight. This step length will be adapted during the movement, depending on the movement pattern, the moving direction (e.g., curve radius) or the recognized environment (e.g., stairs). In the worst case scenario, when the automatic correction doesn't work properly, the user can communicate his current position to the system. Identifying his position by giving information related to his current location (like near a fire extinguisher). For the location of the mobile units to be spread among themselves and to the control and command centers, mechanisms from mobile ad hoc networks and wireless sensor networks are fused to build up a communication network. 
The system has two user interfaces: a conventional one to be used on computer terminals equipped with a screen and a pointing device (mouse, touch screen), and one to be used on wearable computers with head mounted display and speech driven control for action forces.

The system was tested in an outdoor and in an indoor scenario. In the first case the position error equals to a $0.77 \%$ mean value and $0.10 \%$ standard deviation. On the second case the error increases to $2.11 \%$ mean value and $0.95 \%$ standard deviation. This is a good solution, but only for indoor use in buildings. In a forest fire where GPS isn't available, this system doesn't work, since there isn't any CAD drawing plants, and also indoors it only works if CAD drawing exists, which are unfeasible to deploy in all the existing buildings in the world.

Castaneda [6] proposes a shoe-mounted inertial navigation system for pedestrian tracking using a fuzzy logic procedure for better foot stance phase detection, that is applied to the IMU outputs (gyroscope and accelerometer), and an indirect Kalman filter for drift correction based on the typical zero-updating measurement. In its most basic implementation, the shoe-mounted INS estimates and corrects drift errors via an assisted ZVU Kalman filter.

In order to test the system a real time application was developed. The hardware was composed of a laptop PC wire-linked to the IMU. The software consists of the fuzzy logic step detector and an assisted ZVU Indirect Kalman filter. Three different tests were made, involving various walking scenarios composed by forward walks, turns and stairs. The error for each scenario was $30 \mathrm{~cm}, 15 \mathrm{~cm}$, and $27 \mathrm{~cm}$ respectively. Also, positioning the sensors only on the shoes can bring more accuracy errors mainly on the gyroscope, because we move the feet a lot of times to the left or right and not necessarily all the body moves together with them.

The work proposed by [14] presents a system based on modular sensor units, which can be attached to a person and contains various sensors, such as range sensors, inertial and magnetic sensors, a GPS receiver and a barometer. The measurements are processed using Bayesian Recursive Estimation algorithms and combined with available a priori knowledge, such as, map information or human motion models and constraints. Each sensor module contains, apart from its sensors, a low power microprocessor which collects and processes the sensor readings and sends them to the central module.

The current version of the sensor stack consists of an inertial sensor module with a three axis accelerometer, a three axis gyroscope and a three axis magnetometer; and a ranging module which is also used as a wireless data transmission unit and a GPS module. The system is attached to the person's hip area. The inertial and magnetic sensors are used to estimate the heading of the person and to detect walking steps and the corresponding stride length. These steps are detected using a step detection algorithm, which is based on a set of thresholds applied to the vector length of the three accelerometer readings.

A Zero-Velocity-Update method isn't used since they require the sensors to be mounted on the person's feet. However, although the authors state that this method promises a better accuracy, they also claim that it is more feasible to 
wear the sensor unit at the waist, the chest or to hold it in the hand. Like other systems, an indoor map is used as an additional input to the localization algorithm.

While the person is moving through the tracking area, all sensor data are locally preprocessed and then transmitted to a PC for further processing and visualization. Like other systems, the localization is not given in real-time to the user. This is a big limitation if we want to implement a system like the one that we described to help tourists. Most of these systems have this limitation since they use very heavy algorithms that need lots of computational power to process all the retrieved information. One solution can be the inclusion of more sensors to have more information about what is happening on the lower limbs of the user, to reduce the computational power for those algorithms.

Because smartphone's are largely benefiting from the ever increasing rate of integration of mobile IT components, Lukianto et al [16] studied a pedestrian indoor navigation system based on a custom INS and a smartphone. The system is also designed to select the optimal set of auxiliary information from available infrastructure such as WLAN and Bluetooth to calibrate the INS, when necessary. The INS provides a continuous estimate of its current position, speed and orientation. These data are sent to the smartphone to track the current position and for visualization. The smartphone, in turn, collects sensory inputs available to its various communication interfaces and to the (A)GPS receiver system. All the additional sensor information is then processed for plausibility and quality, and used to correct the INS. The updated position is then sent back to the INS.

This INS uses an angular rate sensor, an accelerometer, a magnetic field sensor and a static pressure sensor to obtain the step length. The INS can be connected to the smartphone via Bluetooth or by USB cable. In the future, Kalman filter algorithms will be investigated firstly as a means of INS/GPSintegration, and also the concept of particle filtering will be investigated for viability, providing a means of $2 \mathrm{D}$ localization if a detailed map of the building is available. A big benefit of this system is that it tries to re-use the equipment that the users already have with them (a smartphone) to complement its AGPS module and provide location where the GPS can't. A limitation, is that it only uses one INU, which is not sufficient to provide a good localization accuracy.

The main challenge in this area is to provide an effective position of a person in an environment where GPS is not available and no structured environment exists. To overcome these challenges INS are commonly used, but they have great drift and provide sometimes inaccurate information. In that sense, the next challenge is to reduce these errors so deviations between a virtual trajectory and the real user path can be avoided.

\section{System Overview}

The project that we are entitling as "PLASYS - All Over the Place Location System", aims to study and create a system that, combined with GPS, provides location everywhere, including indoor and dense environments. 
More specifically, this project is divided into three objectives:

- Analyze how activity recognition can be improved (adding different types of sensors) and finding an optimal sensor position on the body;

- Development of methods to process the data acquired from all the sensors, making a sensor fusion; use of probabilistic algorithms to learn the walking/moving behaviors for real-time sensor data correction;

- Expand the existing tourism mobile application to work with the proposed INS.

In order to solve the existing problem, we need to tackle the challenges present in these objectives. In the next sections we will explain each objective, presenting the main challenges and which methodology we will use to tackle them.

\subsection{Sensors}

First of all we will explore the type of sensors to use, since it is intended that, in addition to providing accurate data, they must be also comfortable (and imperceptible) enough to allow their integration into the user's clothing. With this approach we don't need any external sensors, avoiding expensive costs on structured environments.

As stated earlier, an Inertial Navigation System can bring several problems, especially because of the drift that the sensors can have. This is the biggest problem/challenge of the whole project. Also, because of people different sizes, the ideal position of the sensors to one person can be different to another. This leads to another big challenge: the discovery of an ideal spot for the sensors to work in diverse types of persons.

Small sensors will be distributed by the lower limbs (legs and hip area) to collect data. These data will be sent to a central module that will handle the calculations to determine, in real time, the position of an individual. The interconnection of the various modules with the core module will be run through a wireless body network. For now, we don't know which technology, Bluetooth (new version, v4) or ZigBee [13], will be used. The final decision will rely in a series of system tests designed to evaluate which is the most reliable technology.

We pretend to distribute the sensors like this: force sensors and accelerometers in the feet; and a gyroscope and a pressure sensor in the abdominal area (see figure 1).

In the abdominal area, there will be a central module that communicates with a PDA. This PDA will show to the user his current location. The force sensors are essential to determine when the user puts his feet on the ground, that combined with the accelerometer (to get the step acceleration) provides a more exact step length. The gyroscope gets the body travel direction. The pressure sensor on the abdominal area is useful to get the user elevation inside the building.

To have a better perception of the comfortableness of the system a survey research will be done. This type of research method is associated with the use of questionnaires and statistical data for analyze user answers. Using questionnaires is useful to provide a better perception of the proposed system usage. 


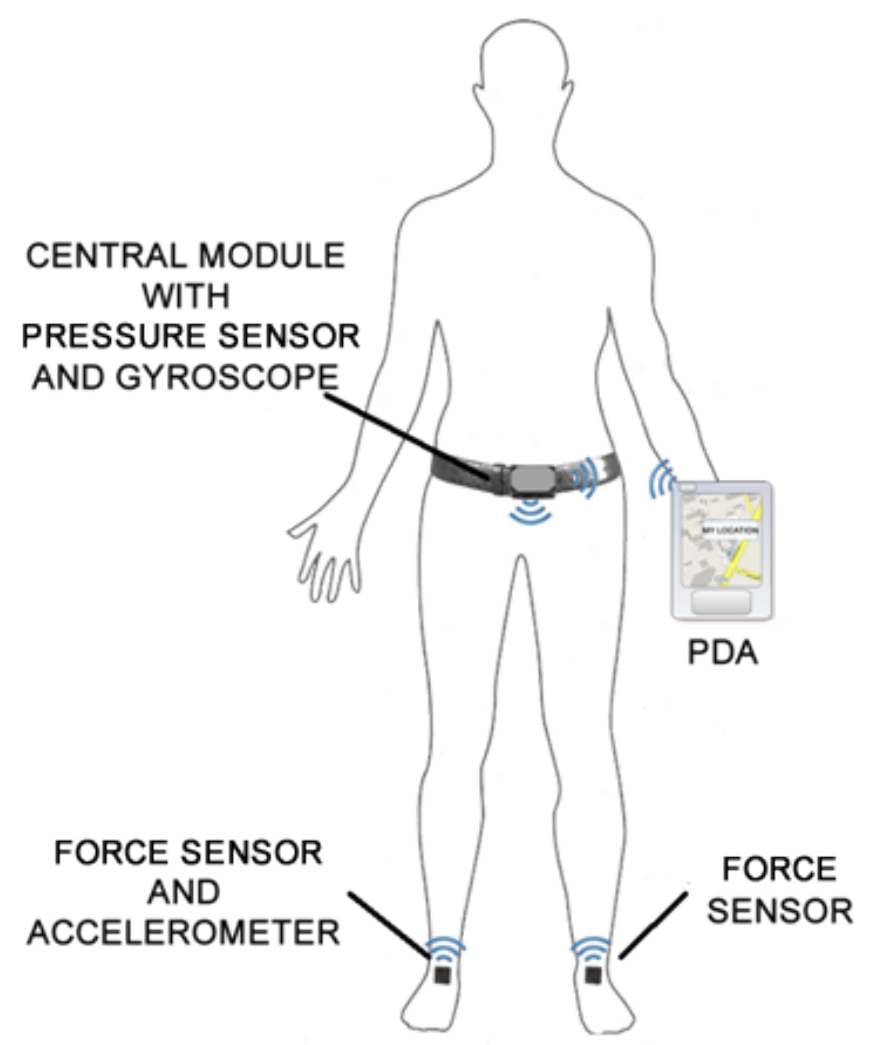

Fig. 1. Disposition of the sensors on the human body

\subsection{Sensor Fusion and Probabilistic Algorithms}

All the sensory set (pressure sensors and accelerometers in the feet, gyroscope and accelerometer in the abdominal area - see figure 1) requires the implementation of a sensor fusion. This sensor fusion will be implemented using models based on Kalman filters.

In order to reduce errors provided by MEMS, heuristic and probabilistic algorithms have to be implemented. They are necessary to make corrections over the provided errors.

These probabilistic algorithms are used to calibrate the system based on GPS. They will work like this, when the user is in environments where the GPS signal is very good, the system can self-learn how the user makes the steps, and the gaps (errors) that exist in those steps. So when the GPS signal is not available, the collected sensor information can be self-corrected by the accuracy algorithms.

As an applicability example for this system, we have a tourist who is on vacation in a city (without the need to have in every building a structured 
location system) and will visit a museum. With this system, he can navigate inside the museum and get information about what he is seeing at the moment (e.g., paintings, sculptures) without other tools besides his PDA. The system, in an outdoor environment learns the pattern of user's stride, and the deviation in sensor readings for future accurate indoor positioning calculation. This is called the training phase, the system first requires a set of data to determine the parameters to detect the walking behaviors. From the training data, the unit motion recognizer automatically extracts parameters such as threshold values and the mean and standard deviations of the sensor signals. The system takes as its starting point the last obtained coordinate from the GPS.

In the future, extensive user experiments will be necessary in order to improve the validity and reliability of the research. These experiments will, not only, allow us to precisely assess how accurate the obtained position of the user is, but also, most importantly, to obtain valuable data about the differences in the step patterns originated from a diverse set of users. This knowledge can give more insight on what the best practices to predict the step length and direction are, and also help the implementation of the automatic calibration of these values.

Another problem that can be originated from all the complexity of the project is the delay between the real location and the processed one (that appears to the user on the mobile device). The process is complex: it starts on the sensors that gather the values, which are afterwards sent to the central module. This process has already communication delays. When the central module has the sensors values, it must combine them according to the data timestamps to process the sensor fusion and estimate the walking path, which takes some processing time. Afterwards, it the probabilistic algorithms are executed to correct the estimated walking path. This algorithm can run for a large amount of time. The algorithms should be efficient to process the data in a short period of time, in order for the delay to be minimized. The last step of the process is the presentation the user's current location on his mobile device. Concluding, as can be imagined, this process with so many steps can bring a big delay on estimating the real location of the user.

After the algorithm implementation, an experiment will be performed. The experiment will focus on investigating the variables and the way in which these can affect the experimental work. It will be used to verify the previously formulated hypothesis. Some experimental walking paths will be created to test the variables of the algorithms and the position of the sensors to see if minor changes can affect the system positively or not.

At least three scenarios, represented on figure 2, will be created. The first one will be as simply as possible, only a straight line with 10 meters, to see if the system can correctly measure the distance (Heavy Dash line on figure 2); The second case will involve straight lines combined with changes of direction (for example, in a building a person must travel through two or more offices) Dash Double line on figure 2; The third one (Dotted line on figure 2) will combine the use of outdoor location with the proposed system: the person is outside of a 


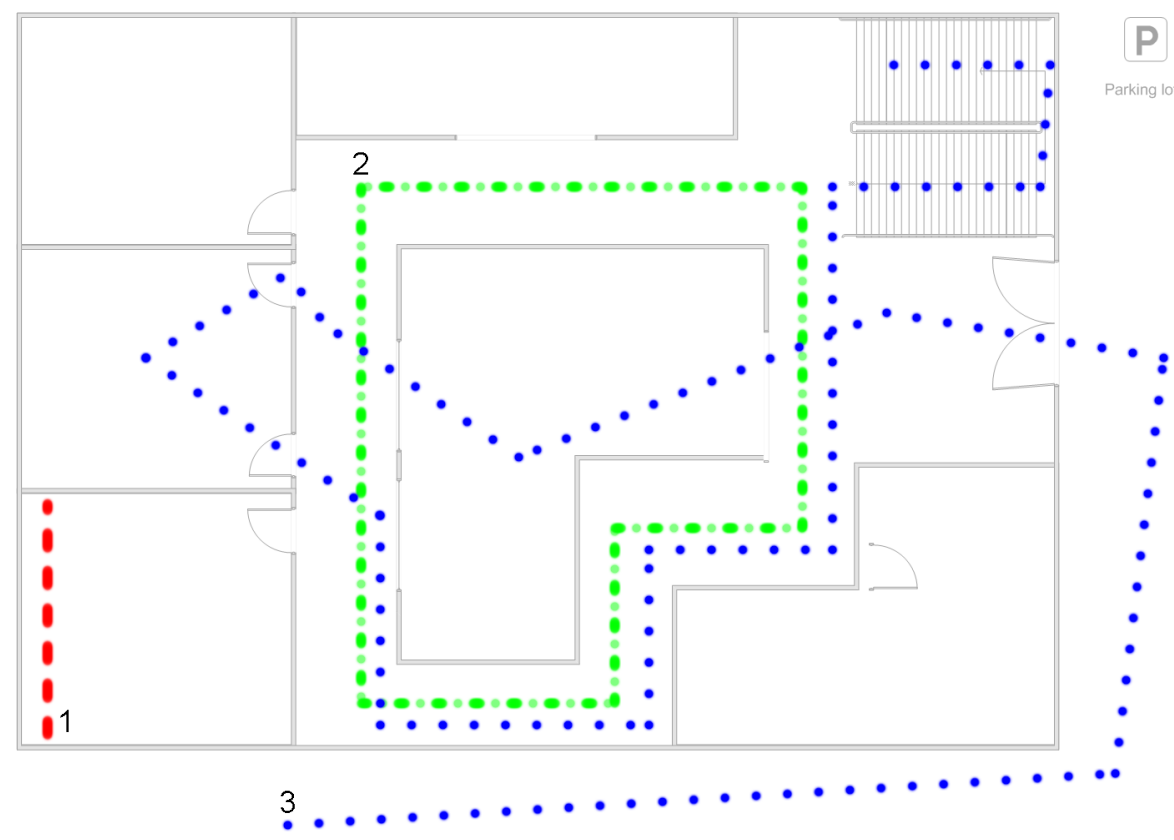

Fig. 2. Experience scenarios - Heavy Dash line (1) represents the first scenario; Dash Double line (2) represents the second scenario; Dotted line (3) represents the third scenario.

building guided by GPS and when he enters the building, the INS must provide the exact location inside the building.

\section{Conclusions}

Retrieving location using an inertial navigation system still remains an open research problem, since there isn't still an approach with a good reliability to use in conjunction with GPS, to retrieve location when GPS is not available. This is a complex problem because of the sensors drift and people's different sizes. This is also a problem when selecting the most appropriate spot to put the sensors.

To tackle these challenges, we propose a system based on probabilistic learning algorithms. The person's walking behavior to predict more effectively the walking path. The project objectives were specified along with the research methods that will be used.

Three quantifiable success criteria were identified to give to the project a success degree. The first one is the accuracy of the estimated location that must be between $90 \%$ and $95 \%$ or, in other words, for each 100 meters traveled the system must have an error of only 5 (to 10) meters. Also, the accumulated error 
must be kept to the minimum possible. The second criterion, is the delay between the readings and presentation to the user of his current location, that must be less than 2 seconds. For higher delay values, positioning will not be considered realtime. The third criterion focuses in the intrusion that the system may have to the people's day-to-day life. This is difficult to measure and not totally quantifiable. However, it is intended for the sensors to be totally wearable and imperceptible to the user. The system should be as easy to use in a point that the user forgets that he's wearing the sensors.

In order to test this system in a real environment with real people, integration with the PSiS system is envisaged, to support tourists on their visits.

\section{References}

1. R. Anacleto, N. Luz, and L. Figueiredo. Personalized sightseeing tours support using mobile devices. IFIP - World Computer Congress 2010, page 301-304, 2010.

2. R. Anacleto, N. Luz, and L. Figueiredo. PSiS mobile. In International Conference on Wireless Networks (ICWN2010), Las Vegas, USA, 2010.

3. P. Bahl and V. N Padmanabhan. RADAR: an in-building RF-based user location and tracking system. In INFOCOM 2000. Nineteenth Annual Joint Conference of the IEEE Computer and Communications Societies. Proceedings. IEEE, volume 2, page $775-784,2000$.

4. S. Beauregard and H. Haas. Pedestrian dead reckoning: A basis for personal positioning. In Proceedings of the 3rd Workshop on Positioning, Navigation and Communication (WPNC'06), page 27-35, 2006.

5. G. Bieber and M. Giersich. Personal mobile navigation systems-design considerations and experiences. Computers $\mathcal{E}$ Graphics, 25(4):563-570, 2001.

6. N. Castaneda and S. Lamy-Perbal. An improved shoe-mounted inertial navigation system. In Indoor Positioning and Indoor Navigation (IPIN), 2010 International Conference on, page 1-6, 2010.

7. J. Elwell. Inertial navigation for the urban warrior. In Proceedings of SPIE, volume 3709, page 196, 1999.

8. F. Evennou and F. Marx. Advanced integration of WiFi and inertial navigation systems for indoor mobile positioning. Eurasip journal on applied signal processing, 2006:164-164, 2006.

9. L. Fang, P. J Antsaklis, L. A Montestruque, M. B McMickell, M. Lemmon, Y. Sun, H. Fang, I. Koutroulis, M. Haenggi, M. Xie, et al. Design of a wireless assisted pedestrian dead reckoning system - the NavMote experience. Instrumentation and Measurement, IEEE Transactions on, 54(6):2342-2358, 2005.

10. H. Hashimoto, K. Magatani, and K. Yanashima. The development of the navigation system for visually impaired persons. In Engineering in Medicine and Biology Society, 2001. Proceedings of the 23rd Annual International Conference of the IEEE, volume 2, page 1481-1483, 2001.

11. J. Hightower and G. Borriello. Location systems for ubiquitous computing. IEEE Computer, 34(8):57-66, 2001.

12. D. Kelly, S. McLoone, and T. Dishongh. A bluetooth-based minimum infrastructure home localisation system. In Wireless Communication Systems, ISWCS'08., page 638-642, 2008.

13. P. Kinney et al. Zigbee technology: Wireless control that simply works. In Communications design conference, volume 2, 2003. 
14. L. Klingbeil, M. Romanovas, P. Schneider, M. Traechtler, and Y. Manoli. A modular and mobile system for indoor localization. In Indoor Positioning and Indoor Navigation (IPIN), 2010 International Conference on, page 1-10, 2010.

15. M. Kourogi, T. Ishikawa, Y. Kameda, J. Ishikawa, K. Aoki, and T. Kurata. Pedestrian dead reckoning and its applications. In Proceedings of "Let's Go Out" Workshop in conjunction with ISMAR, volume 9, 2009.

16. C. Lukianto, C. Honniger, and H. Sternberg. Pedestrian smartphone-based indoor navigation using ultra portable sensory equipment. In Indoor Positioning and Indoor Navigation (IPIN), 2010 International Conference on, page 1-5, 2010.

17. N. Luz, R. Anacleto, and A. Almeida. Tourism mobile and recommendation systems - a state of the art. In International Conference on e-Learning, e-Business, Enterprise Information Systems, and e-Government, Las Vegas, USA, 2010.

18. S. Maskell and N. Gordon. A tutorial on particle filters for on-line nonlinear/nonGaussian bayesian tracking. In Target Tracking: Algorithms and Applications, IEEE, page $2-1,2002$.

19. J. Murray. Wearable computers in battle: recent advances in the land warrior system. In Wearable Computers, 2000. The Fourth International Symposium on, page $169-170,2000$.

20. V. Renaudin, O. Yalak, P. Tomé, and B. Merminod. Indoor navigation of emergency agents. European Journal of Navigation, 5(3):36-45, 2007.

21. H. W Sorenson. Kalman filtering: theory and application. IEEE, 1985.

22. R. Tesoriero, J. Gallud, M. Lozano, and V. Penichet. Using active and passive RFID technology to support indoor location-aware systems. Consumer Electronics, IEEE Transactions on, 54(2):578-583, 2008.

23. Y. K. Thong, M. S. Woolfson, J. A. Crowe, B. R. Hayes-Gill, and R. E. Challis. Dependence of inertial measurements of distance on accelerometer noise. Measurement Science and Technology, 13:1163, 2002.

24. U. Walder, T. Wießflecker, and T. Bernoulli. An indoor positioning system for improved action force command and disaster management. Proceedings of the 6th International ISCRAM Conference, Gothenburg, Sweden, 2009.

25. R. Want, A. Hopper, V. Falcao, and J. Gibbons. The active badge location system. ACM Transactions on Information Systems (TOIS), 10(1):91-102, 1992.

26. O. Woodman and R. Harle. Pedestrian localisation for indoor environments. In Proceedings of the 10th international conference on Ubiquitous computing, page 114-123, 2008.

27. B. E. N. Yue-yang, S. U. N. Feng, G. A. O. Wei, and C. Ming-hui. Study of zero velocity update for inertial navigation [J]. Journal of System Simulation, 17, 2008. 